\title{
Partial Thromboplastin Time
}

National Cancer Institute

\section{Source}

National Cancer Institute. Partial Thromboplastin Time. NCI Thesaurus. Code C38462.

This is a measurement of the intrinsic pathway of coagulation. Significant deficiency in factors II, V, VIII, IX, X, XI, XII or anticoagulants will prolong PTT. (BioT ech Life Science Dictionary) 